\title{
Painful Pes Anserine Bursitis Following Total Knee Replacement Surgery Two cases
}

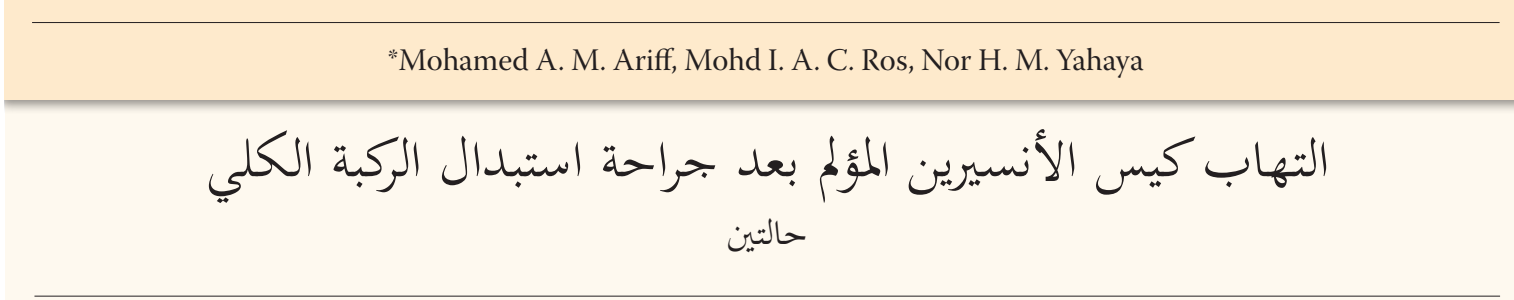

محمد أشراف محمد عارف، محمد عزت أرسلان تشي روس، نور حمدان محمد يحيى

ABSTRACT: Pes anserine bursitis (PAB) is an inflammation of the bursa located between the medial aspect of the tibia and the hamstring muscles. It is common in patients with degenerative or inflammatory knee arthritis, usually has a self-limiting course and tends to respond well to conservative treatment. However, painful PAB directly following total knee replacement surgery is rare. We report two such cases who were diagnosed via ultrasonography at the Universiti Kebangsaan Malaysia Medical Centre, Kuala Lumpur, Malaysia, in 2015. Both patients were treated locally with triamcinolone acetonide under ultrasound guidance and responded well to treatment.

Keywords: Total Knee Replacement; Bursitis; Pain; Case Report; Malaysia.

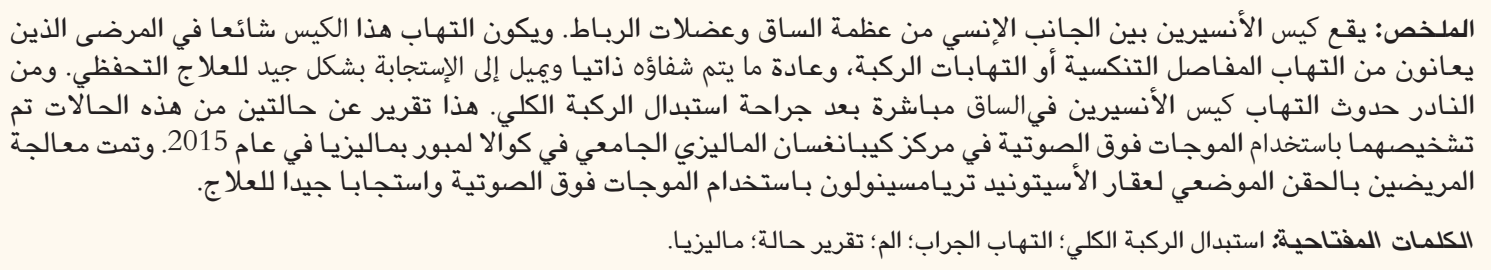

$\mathrm{P}$ ES ANSERINe BURSitis (PAB) IS A SELFlimiting condition whereby the bursa located between the tibia and the three tendons of the hamstring muscle becomes inflammed. ${ }^{1}$ Patients normally present with medialised knee pain which is aggravated upon climbing stairs. The condition has been reported to respond well to exercise and stretching programmes. ${ }^{1}$ The main underlying cause of $\mathrm{PAB}$ is thought to be due to short hamstrings causing a frictional bursal irritation. ${ }^{2}$ Other common causes include direct trauma and degenerative and inflammatory knee diseases. ${ }^{3}$

Generally, PAB as a cause of pain directly following total knee replacement surgery is rare; to the best of the authors' knowledge, only one previous case has been reported which was ultimately found to have been caused by polyethylene wear debris. ${ }^{4}$ This case report describes two patients who developed $\mathrm{PAB}$ following total knee replacement surgeries performed by the same surgeon.

\section{Case One}

A 70-year-old woman with a body mass index (BMI) of $29.6 \mathrm{~kg} / \mathrm{m}^{2}$ presented to the Universiti Kebangsaan Malaysia Medical Centre, Kuala Lumpur, Malaysia, in 2015 for a follow-up appointment. She had previously undergone a total knee replacement surgery in 2014 due to well-controlled rheumatoid arthritis of the left knee joint. At her 12-month follow-up appointment, she complained of a nagging pain in the left medial joint line of her knee which she had first noted shortly after the surgery and which was present only when climbing stairs. She denied any history of falls after the surgery. A physical examination revealed a wellhealed anterior midline longitudinal surgical scar over the left knee. There was no obvious swelling of the area and the range of motion of the knee was 0-120 degrees.

Upon light palpation of the knee, the patient complained of pain extending distally from the left knee medial joint line to a point 3-4 cm medial to the tibial tubercle. There was no obvious erythema or fluctua- 


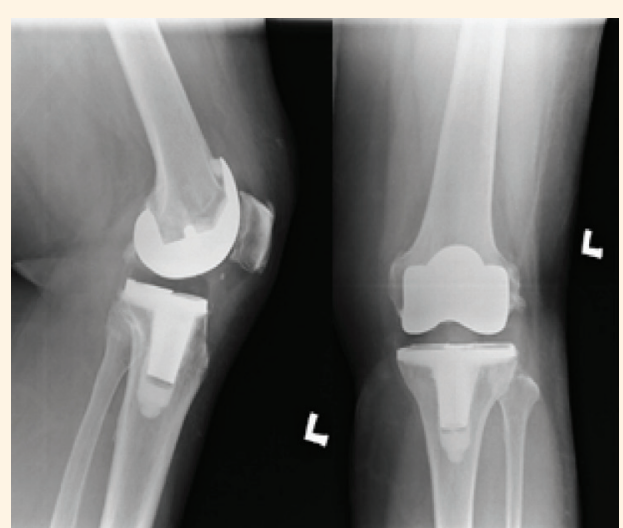

Figure 1: X-ray images of a 70-year-old woman with intermittent pain in the left medial knee joint line following total knee replacement surgery. There was no evidence of osteolysis or loosening of the prosthetic components.

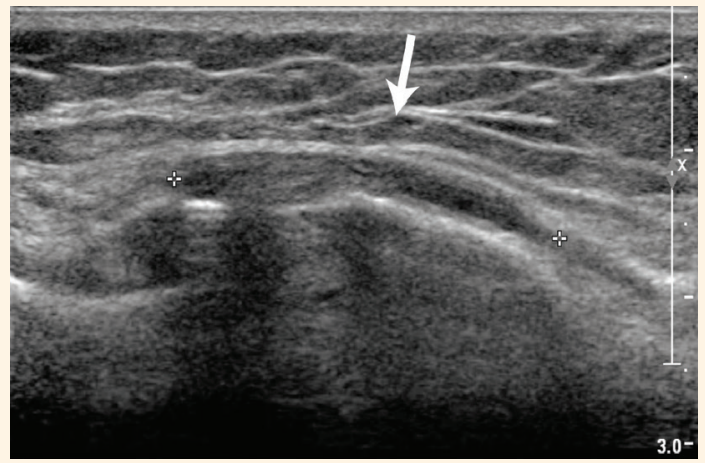

Figure 2: Ultrasound of a 70-year-old woman with intermittent pain in the left medial knee joint line following total knee replacement surgery. Note the thickened appearance of the pes anserinus insertion (arrow).

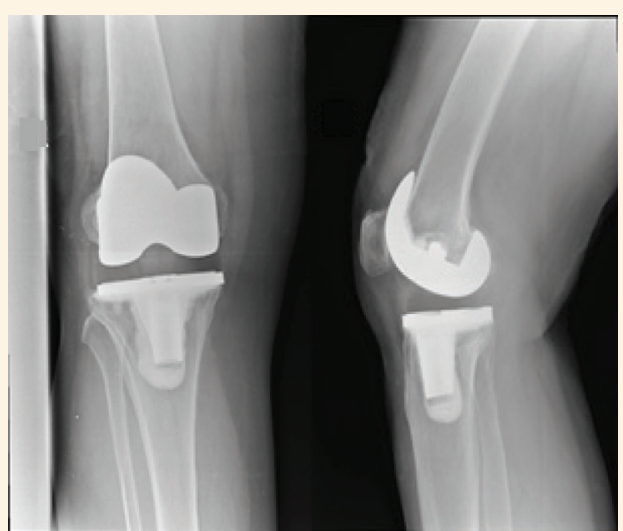

Figure 3: X-ray images of a 77-year-old woman with right-sided medialised knee pain following total knee replacement surgery. No signs of osteolysis or loosening of the prosthetic components could be seen.

tion over the tender area. Radiographs of the knee showed no evidence of osteolysis or loosening of the prosthetic components [Figure 1]. The biomechanical axis of the affected limb appeared well restored. Blood and knee aspirate tests for infective markers did not indicate any signs of infection. An ultrasound showed mild peritendinous effusion of the pes anserinus tendons and thickening of the sheath [Figure 2]. The patient was treated with the local administration of $1 \mathrm{~mL}$ of $40 \mathrm{mg} / \mathrm{mL}$ of triamcinolone acetonide into the bursa under ultrasonography guidance. At periodic three-monthly outpatient follow-up appointments, the patient reported the complete resolution of her symptoms. She continued to be symptom-free three years following the treatment.

\section{Case Two}

A 77-year-old woman with a BMI of $26.4 \mathrm{~kg} / \mathrm{m}^{2}$ presented to the Universiti Kebangsaan Malaysia Medical Centre in 2015 for a follow-up appointment. Eight months earlier, she had undergone a total knee replacement surgery in 2014 due to Kellgren-Lawrence grade 3 osteoarthritis of the right knee. At presentation, she was relatively pain-free except for right-sided medialised knee pain whenever she climbed stairs. On examination, there was a well-healed longitudinal scar anterior to the knee and the range of motion of the knee was $0-130$ degrees. There was pinpoint tenderness and localised swelling over the medial aspect of the knee just below the joint line.

Radiographs showed no evidence of osteolysis or loosening of the prosthetic components and the postoperative biomechanical axis of the limb seemed adequately restored [Figure 3]. As with the former case, a lack of infective blood and knee aspirate markers excluded infection. An ultrasound showed a fluid-filled bursa with peritendinous effusion of the pes anserinus tendons [Figure 4]. A total of $1 \mathrm{~mL}$ of $40 \mathrm{mg} / \mathrm{mL}$ of triamcinolone acetonide was locally administered into the bursa under ultrasonography guidance. Periodic three-monthly outpatient follow-up visits confirmed the complete resolution of the patient's symptoms and there was no evidence of recurrence three years later.

\section{Discussion}

The pes anserinus bursa is one of the 13 bursae around the knee; it is located approximately $5 \mathrm{~cm}$ below the medial knee joint line. ${ }^{5}$ Patients in whom the bursa has a circumscribed anechoic fluid collection of $2 \mathrm{~mm}$ or greater on ultrasonography can be considered to have bursitis. ${ }^{6}$ The inflammation of this conjoined insertion of tendons was first recognised as a unique condition in 1937. ' Most cases of PAB have been reported among patients with underlying degenerative or inflammatory 


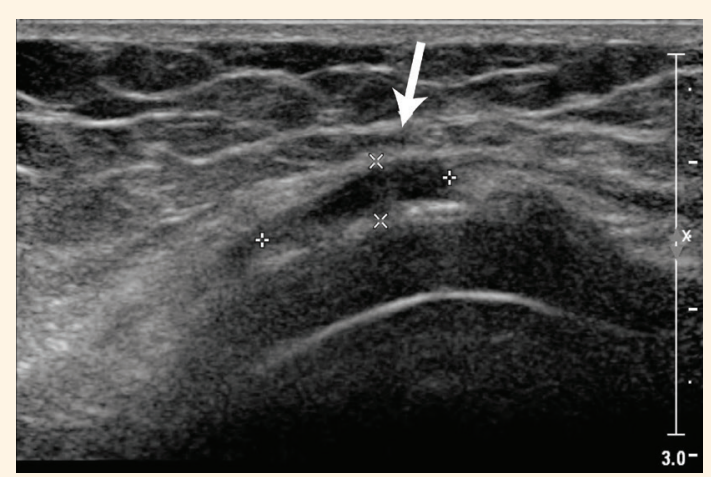

Figure 4: Ultrasound of a 77-year-old woman with right-sided medialised knee pain following total knee replacement surgery. The peritendinous effusion of the pes anserinus tendons with a fluid-filled bursa was noted (arrow).

joint diseases. ${ }^{3}$

In the current case report, both patients were diagnosed with $\mathrm{PAB}$ via ultrasonography. In order to accurately confirm the diagnosis, the affected lower leg was placed in external rotation with the knee flexed at a 30 degree angle. The transducer was first positioned longitudinally relative to the anterior fibres of the medial collateral ligament and then in an oblique transverse position relative to the pes anserinus tendons. In terms of treatment, the ultrasound image was maintained while the triamcinolone acetonide was administered via a $22 \mathrm{G} 38 \mathrm{~mm}$ needle. The needle was introduced through the skin until the needle tip could be visualised between the middle of the pes anserinus and the medial collateral ligament. Ultrasonography was considered preferable to other imaging modalities as it is cost-effective, easily accessible and useful in aiding the administration of the treatment. Ultrasonography-guided injections permit injected substances to be delivered with up to $92 \%$ accuracy. ${ }^{8}$

It is important to note that neither of the two patients reported in the current case report experienced PAB-related symptoms prior to the total knee replacement surgery. Moreover, the researchers were unable to ascertain exactly why these symptoms developed postoperatively. During the total knee replacement, the surgeon used an anterior midline medial parapatellar approach and the same type of implant for both patients. Neither patient had any intraoperative complications and their operative times were not prolonged.

However, at the Universiti Kebangsaan Malaysia Medical Centre, it is common practice to place two Hoffman's retractors on either side of the tibia while making the tibial bone cut with the saw. The retractors allow for good visualisation of the tibia and keep any soft tissue safely away from the saw blades. It is possible that manipulation during the surgery, the placement of the retractors and overzealous retraction may have contributed to the subsequent development of the PAB. Hence, caution should be taken when placing and withdrawing retractors during total knee replacement surgeries, especially on the medial side of the knee.

\section{Conclusion}

Patients who complain of persistent medial knee pain following total knee replacement surgery may have developed PAB. Steroids such as triamcinolone acetonide are a safe and effective way of relieving pain. The use of ultrasonography is recommended as a diagnostic aid as well as to guide the local administration of treatment injections to the affected bursae.

\section{References}

1. Handy JR. Anserine bursitis: A brief review. South Med J 1997; 90:376-7.

2. Rennie WJ, Saifuddin A. Pes anserine bursitis: Incidence in symptomatic knees and clinical presentation. Skeletal Radiol 2005; 34:395-8. doi: 10.1007/s00256-005-0918-7.

3. Larsson LG, Baum J. The syndrome of anserina bursitis: An overlooked diagnosis. Arthritis Rheum 1985; 28:1062-5. doi: 10.10 02/art.1780280915.

4. Huang TW, Wang CJ, Huang SC. Polyethylene-induced pes anserinus bursitis mimicking an infected total knee arthroplasty: A case report and review of the literature. J Arthroplasty 2003; 18:383-6. doi: 10.1054/arth.2003.50062.

5. Chatra PS. Bursae around the knee joints. Indian J Radiol Imaging 2012; 22:27-30. doi: 10.4103/0971-3026.95400.

6. Yoon HS, Kim SE, Suh YR, Seo YI, Kim HA. Correlation between ultrasonographic findings and the response to corticosteroid injection in pes anserinus tendinobursitis syndrome in knee osteoarthritis patients. J Korean Med Sci 2005; 20:109-12. doi: $10.3346 / \mathrm{jkms}$.2005.20.1.109.

7. Moschcowitz E. Bursitis of sartorius bursa: An undescribed malady simulating chronic arthritis. JAMA 1937; 109:1362. doi: 10.1001/jama.1937.92780430001009.

8. Finnoff JT, Nutz DJ, Henning PT, Hollman JH, Smith J. Accuracy of ultrasound-guided versus unguided pes anserinus bursa injections. PM R 2010; 2:732-9. doi: 10.1016/j.pmrj.2010.03.014. 